\title{
Low-Volume Whole-Body Vibration Training Improves Exercise Capacity in Subjects With Mild to Severe COPD
}

\author{
Marc Spielmanns MD, Tobias Boeselt PhD, Rainer Gloeckl PhD, Anja Klutsch, \\ Henrike Fischer MD, Henryk Polanski, Christoph Nell PhD, Jan H Storre PhD MD, \\ Wolfram Windisch PhD MD, and Andreas R Koczulla PhD MD
}

\begin{abstract}
BACKGROUND: The objective of this study was to investigate the benefits of a low-volume out-patient whole-body vibration training (WBVT) program on exercise capacity in comparison with a calisthenics training program in subjects with COPD. METHODS: In this single-center randomized controlled trial, 29 subjects with mild to severe COPD were randomized to WBVT or to calisthenics training, including relaxation and breathing retraining in combination with calisthenics exercises. Both groups equally exercised for a duration of 3 months with 2 sessions of $30 \mathrm{~min} / \mathrm{week}$. Outcome parameters were 6-min walk distance (6MWD, primary outcome), 5-repetition sit-tostand test, leg press peak force, Berg balance scale, St George Respiratory Questionnaire, and COPD assessment test. RESULTS: Twenty-seven subjects completed the study (WBVT, $n=14$; calisthenics training program, $n=13$ ). Baseline characteristics between groups were comparable. Subjects in the WBVT group significantly improved median (interquartile range) 6MWD $(+105$ $[45.5-133.5] \mathrm{m}, P=.001)$, sit-to-stand test $(-2.3[-3.1$ to -1.3$] \mathrm{s}, P=.001)$, peak force $(28.7$ $[16.7-33.3] \mathrm{kg}, P=.001)$, and Berg balance scale $(1.5[0.0-4.0]$ points, $P=.055)$. Changes in 6MWD, sit-to-stand test, and leg press peak force were also found to be significantly different between groups in favor of the WBVT group. Only the between-group difference of the COPD assessment test score was in favor of the calisthenics training group $(P=.02)$. CONCLUSIONS: A low-volume WBVT program resulted in significantly and clinically relevant larger improvements in exercise capacity compared with calisthenics exercises in subjects with mild to severe COPD. (ClinicalTrials.gov registration DRKS9706.) Key words: exercise test; COPD; physical exercise; training. [Respir Care 2017;62(3):315-323. (C) 2017 Daedalus Enterprises]
\end{abstract}

\section{Introduction}

Regular exercise training results in major benefits concerning exercise capacity and quality of life in patients

Dr Spielmanns, Ms Klutsch, and Mr Polanski are affiliated with the Medical Clinic and Pulmonary Rehabilitation in Leverkusen (April), Remigius Hospital, Leverkusen Opladen, Germany. Drs Spielmanns and Windisch are affiliated with the Faculty of Health, Department of Pneumology, University of Witten/Herdecke, Witten, Germany. Drs Boeselt and Koczulla are affiliated with the Department of Medicine, Pulmonary and Critical Care Medicine, Philipps-University Marburg, Marburg, Germany. Dr Gloeckl is affiliated with the Department of Respiratory Medicine and Pulmonary Rehabilitation, Schoen Klinik Berchtesgadener Land, Schoenau am Koenigssee, Germany and the Department of Prevention, Rehabilitation, and Sports Medicine, Klinikum Rechts der Isar, Technical University of Munich, Munich, Germany. Drs Fischer and Nell are affiliated with the Department of Medicine, Training and Health, Philipps- with COPD. ${ }^{1}$ Therefore, guidelines of the Global Initiative for Chronic Obstructive Lung Disease (GOLD) classify exercise training as a cornerstone in disease management. ${ }^{2}$

University Marburg, Marburg, Germany. Drs Storre and Windisch are affiliated with the Department of Pneumology, Cologne Merheim Hospital, Kliniken der Stadt Koeln GmbH, Cologne, Germany. Dr Storre is affiliated with the Department of Pneumology, University Hospital, Freiburg, Germany.

The authors have disclosed no conflicts of interest.

Drs Spielmanns and Boeselt contributed equally to this work.

Correspondence: Marc Spielmanns MD, Medical Clinic and Pulmonary Rehabilitation in Leverkusen (April), Remigius Hospital, Leverkusen Opladen, Germany. E-mail: spielmanns@k-plus.de.

DOI: $10.4187 /$ respcare. 05154 


\section{Low-Volume Whole-Body Vibration in COPD}

The main effects of exercise training are related to improvements in the cardiovascular system as well as skeletal muscle function and morphology. However, the adherence of COPD patients to undergoing and continuing long-term participation in out-patient exercise training programs in general is poor and varies between 10 and 32\%. ${ }^{3}$ New effective exercise training programs of short duration may lead to better acceptance by patients while addressing necessary improvements in patients with COPD.

Whole-body vibration training (WBVT) could be a new and time-saving exercise approach aimed at increasing exercise performance in patients with COPD. In general, WBVT is performed while standing on a vibration platform that applies vibrations at different frequencies. If a subject exercises at high frequencies $(>20 \mathrm{~Hz})$, the muscle relaxation time is interrupted, and that results in a higher output of muscle power. WBVT modalities have been performed successfully in several clinical populations. ${ }^{4}$ Until now, only a few studies have investigated WBVT in subjects with COPD. ${ }^{5-9}$ A reliable comparison of these studies is not possible due to inhomogeneous study protocols and settings, but there seems to be some preliminary evidence that WBVT may be an effective and promising additional exercise method beyond traditional training regimes in patients with COPD. ${ }^{10}$

Calisthenics exercises have been shown to be an effective exercise modality in COPD treatment. ${ }^{11}$ In Germany, out-patient calisthenics exercise groups are well established to maintain long-term effects following pulmonary rehabilitation.

We hypothesized that intensive WBVT of just $1 \mathrm{~h} /$ week may provide a training stimulus sufficient to improve exercise capacity superior to calisthenics training. Therefore, the aim of this study was to compare the effects of a low-volume WBVT for 3 months against conventional calisthenics training in subjects with mild to severe COPD.

\section{Methods}

\section{Setting and Study Design}

A prospective, single-site, randomized controlled trial was performed at St Remigius Hospital (Leverkusen, Germany) in cooperation with Philipps-University Marburg (Marburg, Germany). From August 2015 until January 2016, a total of 50 patients were asked to participate in the study. Twenty-nine subjects were recruited according to the inclusion and exclusion criteria. Subjects were randomized into one of 2 intervention groups, (1) a WBVT group or (2) a calisthenics training group, by using a computer-generated randomization list with a 1:1 ratio (Fig. 1). The study was approved by the ethical board of PhilippsUniversity Marburg (approval 171/14) and was registered at

\section{QUICK LOOK}

\section{Current knowledge}

Comprehensive pulmonary rehabilitation is a very effective and widely recommended therapeutic tool in patients with COPD. A cornerstone of every pulmonary rehabilitation program is an exercise training program particularly addressing endurance and strength. The overall goal is to achieve a significant improvement in physical performance. However, long-term adherence to most pulmonary rehabilitation programs is poor due to time and effort.

\section{What this paper contributes to our knowledge}

A low-volume whole-body vibration training of $1 \mathrm{~h} /$ week can lead to clinically importance and significant improvements in exercise performance compared with calisthenics exercises, and the results are comparable with those of a comprehensive pulmonary rehabilitation program. The vibrating platform can serve as an alternative treatment that provides functional improvement.

the German Clinical Trials Registry (www.germanctr.de; DRKS9706).

\section{Inclusion Criteria}

Subjects were eligible for the study when they fulfilled the following criteria: stable COPD in stage I to III (according to the GOLD classification), ${ }^{2}$ written informed consent, age $\geq 65 \mathrm{y}$, and no involvement in any kind of regular exercise training (at least once per week) within the last 3 months before study inclusion.

\section{Exclusion Criteria}

Patients were not included in the study when they presented one or more of the following issues: clinical signs of a severe cardiac event (eg, congestive heart failure), severe arterial hypertension, severe psychiatric or cognitive impairment, orthopedic comorbidity (eg, artificial joint in the lower extremities), neurological disorders, or an acute COPD exacerbation within the last 4 weeks.

\section{Intervention}

Subjects in the WBVT group trained twice per week for $30 \mathrm{~min}$ for 3 months using a side-alternating vibration platform (Galileo, Novotec Medical, Pforzheim, Germany). WBVT sessions consisted of a warm-up (10 min of treadmill walking or cycling with low intensity, stretching 


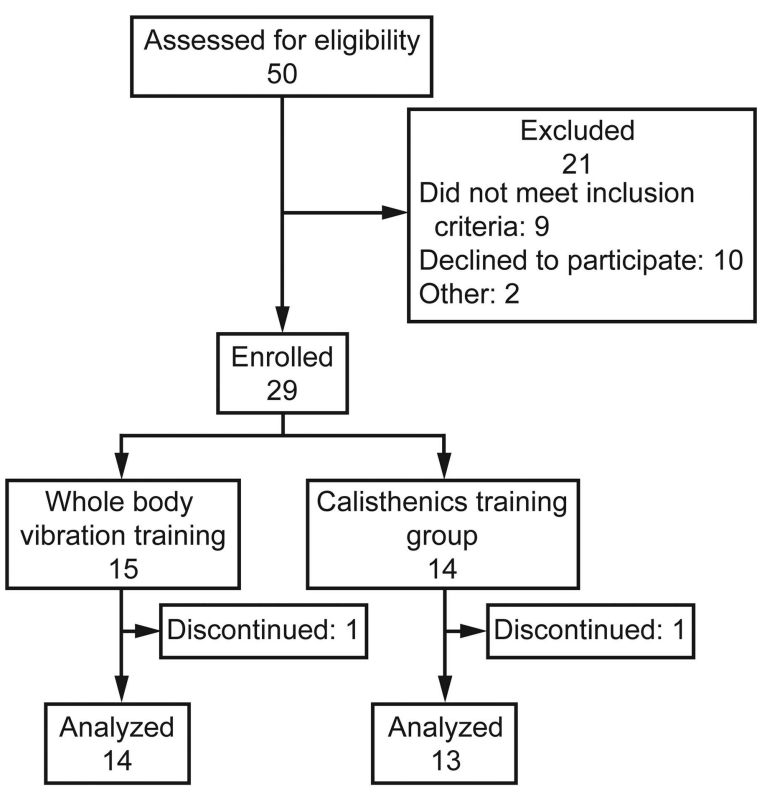

Fig. 1. Flow chart.

exercises), WBVT (15 $\mathrm{min})$, and cool-down (5 $\mathrm{min})$. Training was performed for $3 \times 2 \mathrm{~min}(2 \mathrm{~min}$ of rest in between) at a frequency of $6-10 \mathrm{~Hz}$ and a mean peakto-peak displacement of 4-6 mm during the first 4 weeks. Subjects stood on the WBVT platform shoeless in an isometric squat position with knees slightly bent (knee angle of $\sim 150^{\circ}$ ) and arms by their sides or on the armrest of the device (Fig. 2). During the breaks, subjects were allowed to sit or to walk around as individually preferred. Exercise duration as well as the vibration frequency were progressively increased over time (for WBVT protocol, see Table 1). The calisthenics training group performed group training sessions twice per week for 3 months consisting of $30 \mathrm{~min}$ of relaxation and breathing retraining in combination with calisthenics exercises. Both exercise training groups were supervised by an exercise therapist.

\section{Outcomes}

All measurements were performed at baseline and at the end of the 3-month study period.

6-Minute Walk Test. The primary end point of this study was the difference in the 6-min walk distance (6MWD) from baseline to 3 months between the WBVT and calisthenics training program. For that purpose, a standardized 6-min walk test was performed according to the American Thoracic Society guidelines, ${ }^{12}$ using the reference equation of Troosters et al. ${ }^{13}$

Lung Function Testing. Spirometry and body plethysmography were performed in accordance with the guide-

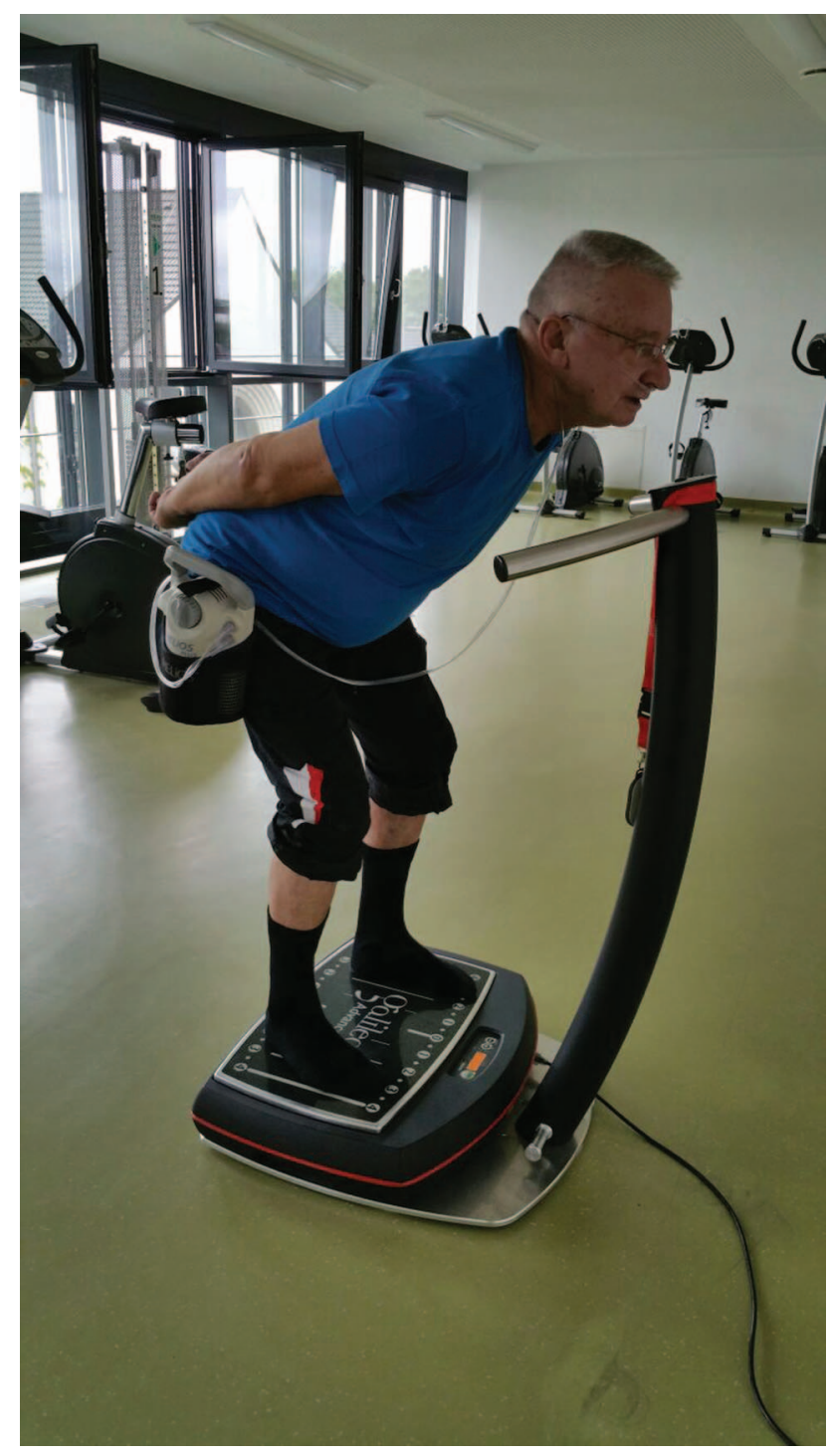

Fig. 2. Subject during whole-body vibration training. With permission.

lines of the American Thoracic Society ${ }^{14,15}$ and reference values from the European Respiratory Society. ${ }^{16}$

5-Repetition Sit-to-Stand Test. The 5-repetition sit-tostand test was performed using a chair with a standard height of $46 \mathrm{~cm}$. Subjects were asked to stand up and sit down 5 times as quickly as possible with their arms folded across their chest. During the standing position, knees and hips had to be fully extended. The outcome of this test was the time needed to complete the test. ${ }^{17}$

Leg Press Peak Force. The one-repetition maximum of the lower extremities was determined by using a leg press device (Funktionsstemme Reha, MedLife, Gallbrunn, Austria). The one-repetition maximum was calculated by using tables for determining the theoretical peak force. ${ }^{18}$ The 
Table 1. Training Program for Whole-Body Vibration Training Group

\begin{tabular}{|c|c|c|c|}
\hline & Weeks $1-4$ & Weeks 5-8 & Weeks 9-12 \\
\hline Training session & $\begin{array}{l}\text { Twice/week for } 3 \times 2 \mathrm{~min}, 2 \text {-min } \\
\text { rest in between }\end{array}$ & $\begin{array}{l}\text { Twice/week for } 3 \times 3 \mathrm{~min}, 2 \text {-min } \\
\text { rest in between }\end{array}$ & $\begin{array}{l}\text { Twice/week for } 3 \times 3 \mathrm{~min}, 2 \text {-min } \\
\text { rest in between }\end{array}$ \\
\hline Aim of WBVT & Muscle relaxation, proprioception & Improve intermuscular coordination & Improve muscle power and strength \\
\hline WBVT frequency, $\mathrm{Hz}$ & $6-10$ & $12-18$ & $21-24$ \\
\hline
\end{tabular}

equation used for maximal repetitions from 1 to 10 was: One-repetition maximum $=($ weight lifted $) /[1.0278-($ repetitions $\times 0.0278)]$. For example, for a subject can benchpress $70 \mathrm{~kg} 6$ times, the estimate of his one-repetition maximum using the equation above is: One-repetition maximum $=70 /[1.0278-(6 \times 0.0278)]=70 /(1.0278-$ $0.1668)=70 / 0.861=81.3 \mathrm{~kg}$.

Muscle Cross-Sectional Area. The muscle cross-sectional areas of the quadriceps rectus femoris and quadriceps intermedius were measured by ultrasound at predefined points of the thigh. ${ }^{19}$

Berg Balance Scale. The Berg balance scale consists of 14 different tasks (eg, standing up from a chair, standing on one leg, picking up an item from the ground) and assesses static and dynamic balance performance. ${ }^{20}$ The total Berg balance scale assessment takes $\sim 15 \mathrm{~min}$. The investigator scores each task between 0 (failed to perform the task) and 4 points (task was performed without any problems). All scores are then summarized, yielding a maximum score of 56 points (= excellent balance performance). The Berg balance scale is a well-validated tool to assess subjects' risk of falling. ${ }^{21}$

Single-Leg Stance. The single-leg stance test provides a quantitative measurement of postural control. ${ }^{22}$ Subjects are asked to stand on one leg as long as possible without contacting the floor with the other leg. Time until task failure is taken as the outcome parameter of this test.

St George Respiratory Questionnaire. The St George Respiratory Questionnaire is a multidimensional questionnaire that assesses disease-specific health-related quality of life in subjects with chronic lung diseases. The St George Respiratory Questionnaire is a self-administered questionnaire with 50 items comprising 3 domains (symptoms, activity, and impact), each scored from 0 to 100 , with higher scores corresponding to worse health-related quality of life. ${ }^{23}$

COPD Assessment Test. This COPD-specific questionnaire evaluates the overall burden in subjects with COPD using 8 different questions. Each question can be scored from 0 points (not impaired) to 5 points (maximally impaired). Accordingly, the total score can range from 0 to 40 points, with higher scores reflecting greater burden of the disease. ${ }^{24}$ The most reliable estimate of the minimum important difference of the COPD assessment test is 2 points. ${ }^{25}$

Statistical Analyses. The sample size calculation was performed using $\mathrm{G}^{*}$ Power 3.1 and yielded a total sample size of 18 subjects considering 2 degrees of freedom with a power $(1-\beta)$ of $95 \%$ and an $\alpha$ of $5 \% .^{26}$

The results are given as the median with interquartile range. Delta values were calculated for each parameter between before (baseline) and after (3 months) measurements. Statistical analyses for between-group comparisons were performed using the Mann-Whitney U test. Comparisons between baseline parameters were done by chi-square test (for frequency distributions) or the U test. Intragroup differences were compared using the Wilcoxon signedrank test. All statistical tests were performed 2-sided with a significance level of $\alpha$ at $5 \%$.

\section{Results}

\section{Baseline Characteristics}

Twenty-seven of 29 subjects (WBVT, $n=14$; calisthenics training program, $n=13$ ) completed the study and were included in the final per-protocol analysis. For dropout reasons, see Figure 1. Baseline characteristics (Table 2) were only significantly different concerning the sit-tostand test. All other parameters were comparable between groups.

\section{Exercise Capacity}

Subjects in the WBVT group were able to increase 6MWD by 105 (45.5-133.5) $\mathrm{m}$ ( $P=.001$; Table 3$)$. The improvement between groups was significantly $(P=.001)$ higher compared with the calisthenics training group (15 [ -3 to 21$] \mathrm{m}, P=.10$ ) (Fig. 3). Also, during the 5-repetition sit-to-stand test, only subjects in the WBVT group 
Table 2. Baseline Characteristics

\begin{tabular}{|c|c|c|c|}
\hline Characteristics & WBVT $(n=14)$ & CTG $(n=13)$ & $P$ \\
\hline Male/female sex, $n$ & $7 / 7$ & $7 / 6$ & .84 \\
\hline Age, median (IQR) y & $69(65.3-73)$ & $70.0(66-78)$ & .34 \\
\hline COPD I/II/III, $n$ & $3 / 6 / 5$ & $3 / 4 / 6$ & .80 \\
\hline Pack-years, median (IQR) & $35.0(9.8-45)$ & $23.0(10-30)$ & .53 \\
\hline BMI, median (IQR) kg/m² & $27.2(24.6-34)$ & $30.6(29-32.5)$ & .38 \\
\hline $\mathrm{FEV}_{1}$, median (IQR) L & $1.5(1-1.8)$ & $1.5(1.4-1.7)$ & .65 \\
\hline $\mathrm{FEV}_{1}$, median (IQR) $\%$ predicted & $63.0(39.3-71)$ & $52.0(43-73)$ & .85 \\
\hline Tiffeneau index, median (IQR) \% & $57.5(38-64.8)$ & $63.0(54-67)$ & .38 \\
\hline 6MWD, median (IQR) m & $507.0(439.8-594.8)$ & $490.0(410-608)$ & .56 \\
\hline 5-Rep STST, median (IQR) s & $12.9(11.6-13.9)$ & $15.0(14-16.3)$ & .035 \\
\hline Leg press peak force, median (IQR) $\mathrm{kg}$ & $92.0(66.7-138)$ & $130.0(112-142)$ & .12 \\
\hline Arterial hypertension, $n(\%)$ & $6(43)$ & $4(31)$ & .52 \\
\hline Diabetes mellitus, $n(\%)$ & $2(14)$ & $3(23)$ & .56 \\
\hline Cancer, $n(\%)$ & $2(14)$ & $3(23)$ & .56 \\
\hline Cardiovascular diseases, $n(\%)$ & $4(28)$ & $2(15)$ & .41 \\
\hline $\begin{array}{l}\text { WBVT }=\text { whole-body vibration training } \\
\text { CTG = calisthenics training group } \\
\text { IQR }=\text { interquartile range } \\
\text { BMI = body mass index } \\
\text { 6MWD }=6 \text {-min walk distance } \\
\text { 5-Rep STST = 5-repetition sit-to-stand test }\end{array}$ & & & \\
\hline
\end{tabular}

could significantly improve testing time by $-2.3(-3.1$ to -1.3) s $(P=.001)$, whereas sit-to-stand test time for subjects in the calisthenics training group remained unchanged (Fig. 4). The same was true for changes in leg press peak force (WBVT: 28.7 [16.7 to 33.3$] \mathrm{kg}, P=.001$ vs calisthenics training program: $3.0[-2.5$ to 5.8$] \mathrm{kg}, P=.13$; Fig. 5).

No significant intra- or intergroup differences were found for the single-leg stance test. However, the multidimensional Berg balance scale improved significantly by 1.5 (0.0-4.0) points $(P=.053)$ only in the WBVT group. Measurements of the cross-sectional area of the rectus femoris or the quadriceps intermedius did not yield any significant changes in any group.

\section{Health-Related Quality of Life}

Changes in the different St George Respiratory Questionnaire domains did not reach significance. However, the between-group difference of the COPD assessment test score was significantly $(P=.02)$ larger in favor of the calisthenics training group (Table 4$)$. No adverse events were observed during the study period.

\section{Discussion}

Our study shows significantly larger improvements in exercise capacity during a 3-month WBVT intervention in subjects with COPD than can be achieved with calisthen- ics exercises. Such large effects on exercise capacity are usually only reported after more comprehensive pulmonary rehabilitation programs. ${ }^{27-29}$ It is remarkable that subjects reached these improvements despite their high exercise capacity at baseline and with a time investment of only $1 \mathrm{~h}$ /week. It is known that patients with COPD, especially those with major limitations in the 6MWD at the beginning of the program, benefit the most from the exercise training. ${ }^{30}$ However, the minimum important difference of $30 \mathrm{~m}$ in the 6MWD was clearly exceeded in the current study. ${ }^{12}$ This finding is in line with previous WBVT studies that also showed beneficial effects in subjects with COPD but using different more time-consuming study settings and designs. 5-7,31

A study published by Pleguezuelos et $\mathrm{al}^{6}$ investigated the effects of WBVT versus usual care in 51 subjects with COPD (GOLD stages III and IV). After 6 weeks of training (3 times/week), subjects in the WBVT group increased 6MWD by $80 \mathrm{~m}$. This finding is in line with our results. However, there are some substantial differences between these 2 studies. Subjects in GOLD stages I to III were included in our study. Accordingly, these subjects were less impaired in their functional exercise capacity (6MWD at baseline, $510 \mathrm{~m}$ ) compared with subjects in the Pleguezuelos study al ${ }^{6}$ (6MWD at baseline, $410 \mathrm{~m}$ ). It is known that subjects with lower exercise capacity respond better to exercise training than subjects with a normal exercise capacity. ${ }^{32}$ Interestingly, the current study demonstrated that also subjects with mild to moderate disease and well pre- 


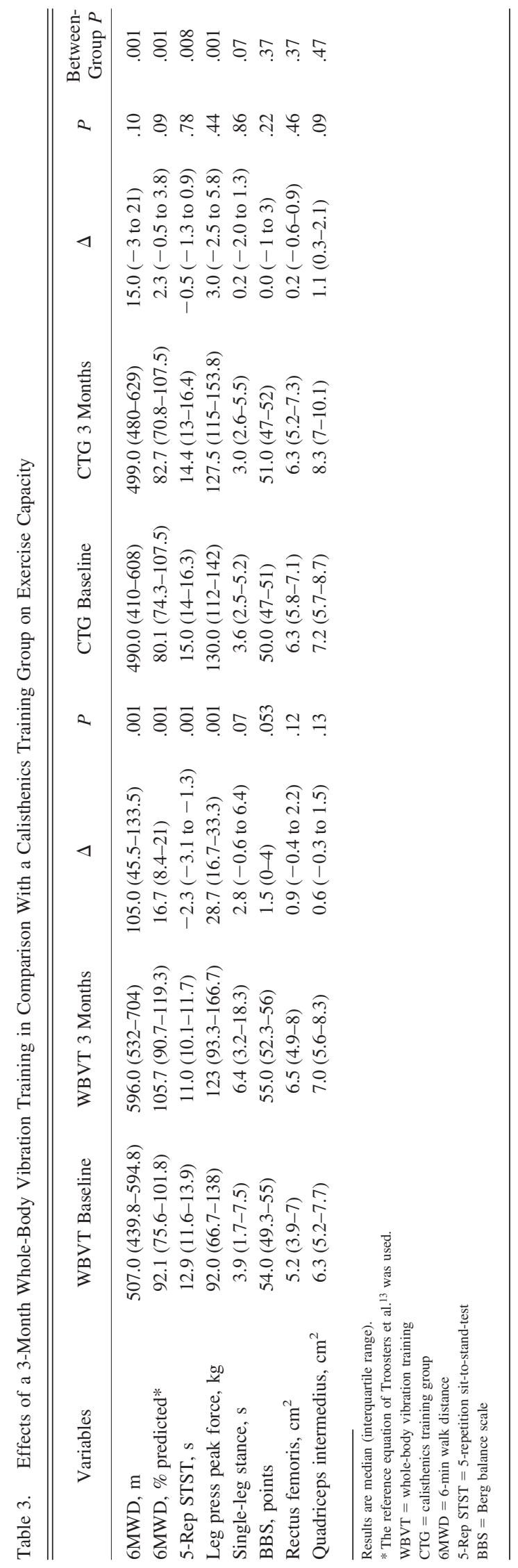

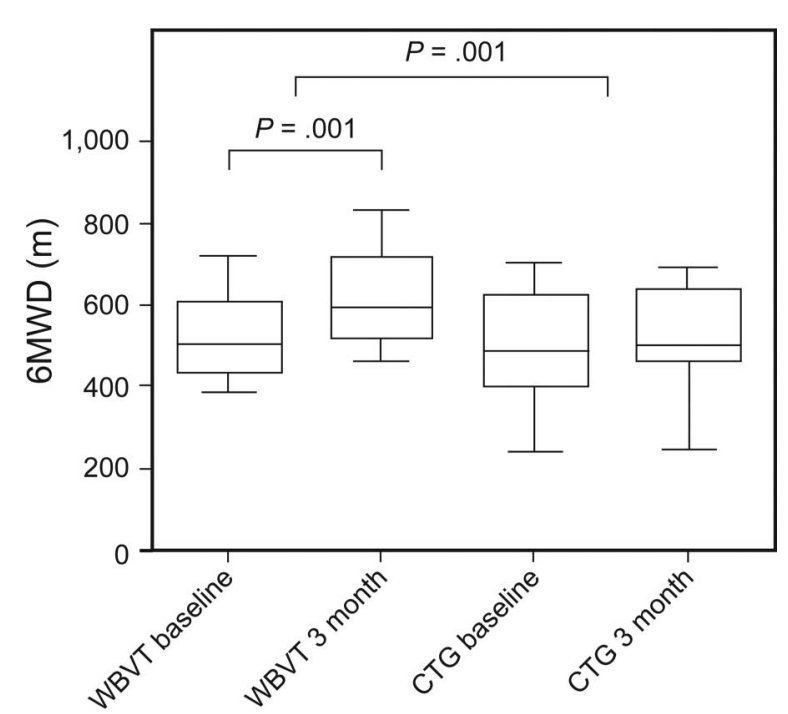

Fig. 3. Box and whisker plot of whole-body vibration training (WBVT) compared with calisthenics training group (CTG) (baseline vs after 3 months) for 6 -min walk distance (6MWD). Boxes represent 25th to 75 th percentiles, center lines denote the median, and whiskers show minimum and maximum values.

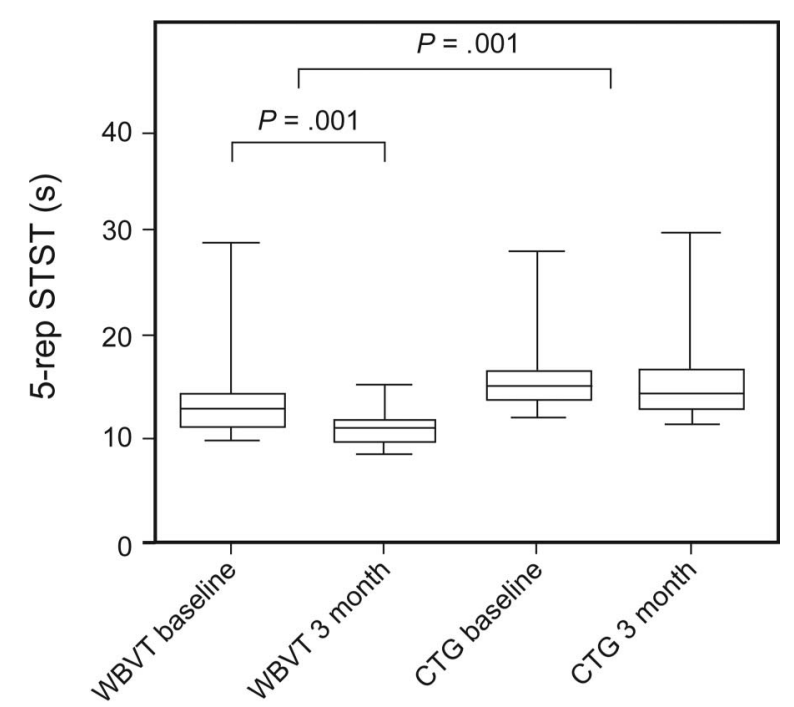

Fig. 4. Box and whisker plots of whole-body vibration training (WBVT) compared with the calisthenics training group (CTG) (baseline vs after 3 months) in the 5-repetition sit-to-stand-test (5-rep STST). Boxes represent 25th to 75th percentiles, center lines denote the median, and whiskers show minimum and maximum values.

served exercise capacity can benefit from WBVT ( $\triangle 6 \mathrm{MWD}, 105 \mathrm{~m})$. Furthermore, only men were included, and the control group in the Pleguezuelos trial $\mathrm{al}^{6}$ performed no active intervention, whereas the control group in our trial received an active training program. This emphasizes the value of WBVT, since between-group differences might be expected to be smaller when comparing 2 active interventions. 


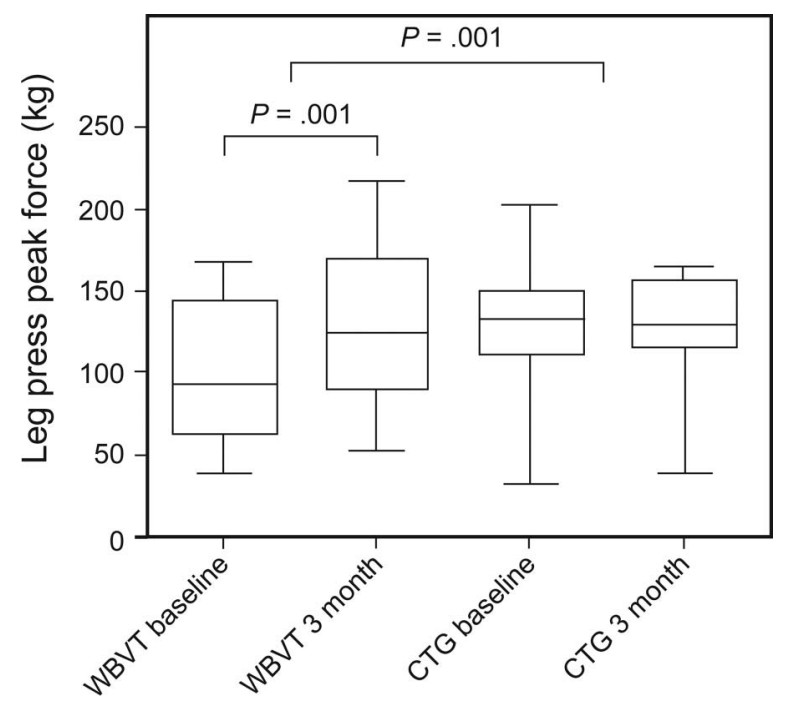

Fig. 5. Box and whisker plot of whole body vibration training (WBVT) compared with the calisthenics training group (CTG) (baseline vs after 3 months) for leg press peak force. Boxes represent 25th to 75th percentiles, center lines denote the median, and whiskers show minimum and maximum values.

Also, the subjects' adherence concerning WBVT in the current study was very high (dropout rate of 7\%), although the training intensity was progressively increased. This is remarkable because out-patient WBVT training studies in subjects with COPD usually report dropout rates of $12-73 \%, 33$ and exercise training programs in general report dropout rates of $10-32 \% .{ }^{3}$ One possible reason for the low dropout rate in the current study could be the short duration of the training sessions (twice weekly for $30 \mathrm{~min}$ ), and maybe participants tended to have positive expectations about the benefits of exercise in the future. In the Pleguezuelos et al trial ${ }^{6}$ the WBVT training intensity chosen was very high $(34 \mathrm{~Hz})$ in comparison with our protocol (maximum $24 \mathrm{~Hz}$ in the last 4 weeks). The drop out rate was also much higher (15\%), although the study duration was half (6 weeks vs 12 weeks).

Patients with COPD often present impaired postural control compared with healthy age-matched patients. Moreover, recent studies suggest that these subjects frequently fall. ${ }^{31,32}$ Associated factors contributing to impaired postural control are muscle weakness, physical inactivity, the need for supplemental oxygen, and limited mobility. ${ }^{34}$ Former studies have shown that WBVT positively impacts posture and balance in the healthy elderly. ${ }^{35}$ However, up to now, the effects of WBVT on balance in subjects with COPD have not yet been investigated. Our study has shown that subjects in the WBVT were able to significantly improve balance (as measured by the Berg balance scale), but the betweengroup difference in the Berg balance scale was not significantly different. However, it must be considered that

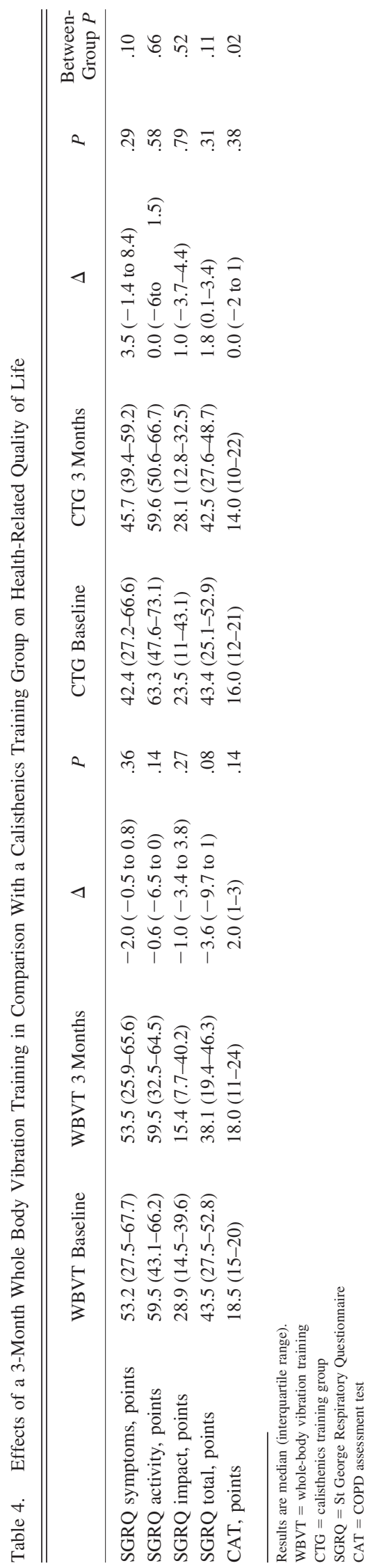




\section{Low-Volume Whole-Body Vibration in COPD}

subjects in the WBVT group reached a baseline value of 52 points out of a maximum of 56 points in this test. This high baseline value might have contributed to a ceiling effect. Also the large between-group difference at baseline for the single-leg stance (11 s for the WBVT group vs $5 \mathrm{~s}$ for the calisthenics training group) showed that subjects in the WBVT group had a better balance capacity at baseline.

Measurements of the muscle cross-sectional area did not yield any significant changes in the 2 groups. It seems that the improvements in exercise capacity induced by WBVT may not be explained by a measurable increase in muscle mass of lower extremities. In general, the application of mechanical vibrations to the human body induces a mechanical stress on different kinds of tissue (eg, bones, muscles, skin, or nerves). This vibration elicits involuntary muscle contraction and relaxation circles (induced by muscle stretch reflex). Therefore, the benefits of WBVT are more related to neurophysiological improvements than to muscle hypertrophy. ${ }^{36-38}$ Although the WBVT frequency in this study was increased up to $24 \mathrm{~Hz}$ (high intensity), the load on the muscles might have been still too low to achieve muscle hypertrophy. WBVT might have been sufficient to increase muscle mass had additional weights been used. ${ }^{39}$

COPD impact on health status as measured by the COPD assessment test increased significantly only in the calisthenics training group. But the difference was not of clinical relevance, since the minimum important difference of 2 points was not exceeded. ${ }^{25} \mathrm{We}$ assume that the COPD assessment test might not be specific enough to detect changes in exercise capacity, since only 2 of 8 questions of the COPD assessment test are related to physical activity. COPD severity assessed by the COPD assessment test score can be directly related to air-flow limitation. ${ }^{40}$ That there were no changes in the COPD assessment test score is not surprising, since we observed no changes in air-flow obstruction in our subjects. However, only subjects in the WBVT group increased quality of life measured by the more sensitive multidimensional St George Respiratory Questionnaire, but this nonsignificant increase was under the estimated minimum important difference threshold of -4 points. ${ }^{41}$

Our study also has some limitations that should be considered. The number of participants was quite low. However, it was still above the a priori calculated sample size. Also, some outcome parameters like the single-leg stance or the COPD assessment test might have been rather insensitive tools to assess balance or quality of life. Other assessment tests with a higher discriminatory power would have been useful. Nevertheless, these assessment tests were only secondary outcome parameters.

\section{Conclusions}

In summary, our study showed that in cases of mild to severe COPD, a low-volume WBVT program can result in significant and clinically relevant larger improvements in exercise capacity than can be achieved with calisthenics exercises. We assume that a short training of only $1 \mathrm{~h} /$ week will result in better training discipline and a lower dropout rate over a period of 3 months.

\section{REFERENCES}

1. Foglio K, Bianchi L, Bruletti G, Porta R, Vitacca M, Balbi B, Ambrosino N. Seven-year time course of lung function, symptoms, healthrelated quality of life, and exercise tolerance in COPD patients undergoing pulmonary rehabilitation programs. Respir Med 2007; 101(9):1961-1970.

2. Global Initiative for Chronic Obstructive Lung Disease (GOLD). Global Strategy of the diagnosis, management, and prevention of chronic obstructive pulmonary disease. Updated 2016. www.goldcopd. org/global-strategy-diagnosis-management-prevention-copd-2016.

3. Keating A, Lee A, Holland AE. What prevents people with chronic obstructive pulmonary disease from attending pulmonary rehabilitation? A systematic review. Chron Respir Dis 2011;8(2):89-99.

4. Blottner D, Salanova M, Püttmann B, Schiffl G, Felsenberg D, Buehring B, Rittweger J. Human skeletal muscle structure and function preserved by vibration muscle exercise following 55 days of bed rest. Eur J Appl Physiol 2006;97(3):261-271.

5. Gloeckl R, Heinzelmann I, Baeuerle S, Damm E, Schwedhelm AL, Diril $M$, et al. Effects of whole body vibration in patients with chronic obstructive pulmonary disease: a randomized controlled trial. Respir Med 2012;106(1):75-83.

6. Pleguezuelos E, Pérez ME, Guirao L, Samitier B, Costea M, Ortega $\mathrm{P}$, et al. Effects of whole body vibration training in patients with severe chronic obstructive pulmonary disease. Respirology 2013; 18(6):1028-1034.

7. Salhi B, Malfait TJ, Van Maele G, Joos G, van Meerbeeck JP, Derom E. Effects of whole body vibration in patients with COPD. COPD 2015;12(5):525-532.

8. Boeselt T, Nell C, Kehr K, Holland A, Dresel M, Greulich T, et al. Whole-body vibration therapy in intensive care patients: a feasibility and safety study. J Rehabil Med 2016;48(3):316-321.

9. Greulich T, Nell C, Koepke J, Fechtel J, Franke M, Schmeck B, et al. Benefits of whole body vibration training in patients hospitalised for COPD exacerbations: a randomized clinical trial. BMC Pulm Med 2014;14:60.

10. Gloeckl R, Heinzelmann I, Kenn K. Whole body vibration training in patients with COPD: a systematic review. Chron Respir Dis 2015; 12(3):212-221.

11. Spielmanns M, Göhl O, Schultz K, Worth H. Lung exercise: outpatient exercise program has long-term benefits on COPD. Dtsch Med Wochenschr 2015;140(13):1001-1005.

12. Holland AE, Spruit MA, Troosters T, Puhan MA, Pepin V, Saey D, et al. An official European Respiratory Society/American Thoracic Society technical standard: field walking tests in chronic respiratory disease. Eur Respir J 2014;44(6):1428-1446.

13. Troosters T, Gosselink R, Decramer M. Six minute walking distance in healthy elderly subjects. Eur Respir J 1999;14(2):270-274.

14. Miller MR, Hankinson J, Brusasco V, Burgos F, Casaburi R, Coates A, et al. Standardisation of spirometry. Eur Respir J 2005;26(2):319338 . 


\section{Low-Volume Whole-Body Vibration in COPD}

15. Wanger J, Clausen JL, Coates A, Pedersen OF, Brusasco V, Burgos $\mathrm{F}$, et al. Standardisation of the measurement of lung volumes. Eur Respir J 2005;26(3):511-522.

16. Quanjer PH, Tammeling GJ, Cotes JE, Pedersen OF, Peslin R, Yernault JC. Lung volumes and forced ventilatory flows: report working party standardization of lung function tests, European Community for Steel and Coal: official statement of the European Respiratory Society. Eur Respir J Suppl 1993;16:5-40.

17. Jones SE, Kon SS, Canavan JL, Patel MS, Clark AL, Nolan CM, et al. The five-repetition sit-to-stand test as a functional outcome measure in COPD. Thorax 2013;68(11):1015-1020.

18. Heyward VH. Advanced fitness assessment and exercise prescription, 2nd edition. Champaign, Illinois: Human Kinetics Books; 1991: 106-108.

19. Seymour JM, Ward K, Sidhu PS, Puthucheary Z, Steier J, Jolley CJ, et al. Ultrasound measurement of rectus femoris cross-sectional area and the relationship with quadriceps strength in COPD. Thorax 2009; 64(5):418-423.

20. Wong CK, Chen CC, Blackwell WM, Rahal RT, Benoy SA. Balance ability measured with the Berg balance scale: a determinant of fall history in community-dwelling adults with leg amputation. J Rehabil Med 2015;47(1):80-86.

21. Blum L, Korner-Bitensky N. Usefulness of the Berg Balance Scale in stroke rehabilitation: a systematic review. Phys Ther 2008;88(5): 559-566.

22. Trojian TH, McKeag DB. Single leg balance test to identify risk of ankle sprains. Br J Sports Med 2006;40(7):610-613; discussion 613.

23. Jones PW, Quirk FH, Baveystock CM. The St George's Respiratory Questionnaire. Respir Med 1991;85(Suppl B):25-31; discussion 3337.

24. Jones PW, Harding G, Berry P, Wiklund I, Chen WH, Kline Leidy N. Development and first validation of the COPD Assessment Test. Eur Respir J 2009;34(3):648-654.

25. Kon SS, Canavan JL, Jones SE, Nolan CM, Clark AL, Dickson MJ, et al. Minimum clinically important difference for the COPD Assessment Test: a prospective analysis. Lancet Respir Med 2014;2(3): 195-203.

26. Faul F, Erdfelder E, Lang AG, Buchner A. G*Power 3: a flexible statistical power analysis program for the social, behavioral, and biomedical sciences. Behav Res Methods 2007;39(2):175-191.

27. Revitt O, Sewell L, Morgan MD, Steiner M, Singh S. Short outpatient pulmonary rehabilitation programme reduces readmission following a hospitalization for an exacerbation of chronic obstructive pulmonary disease. Respirology 2013;18(7):1063-1068.

28. Borghi-Silva A, Mendes RG, Trimer R, Oliveira CR, Fregonezi GA, Resqueti VR, et al. Potential effect of 6 versus 12-weeks of physical training on cardiac autonomic function and exercise capacity in chronic obstructive pulmonary disease. Eur J Phys Rehabil Med 2015;51(2):211-221.
29. Amin S, Abrazado M, Quinn M, Storer TW, Tseng CH, Cooper CB. A controlled study of community-based exercise training in patients with moderate COPD. BMC Pulm Med 2014;14:125.

30. Spielmanns M, Gloeckl R, Schmoor C, Windisch W, Storre JH, Boensch M, Kenn K. Effects on pulmonary rehabilitation in patients with COPD or ILD: a retrospective analysis of clinical and functional predictors with particular emphasis on gender. Respir Med 2016;113:8-14.

31. Braz Júnior DS, Dornelas de Andrade A, Teixeira AS, Cavalcanti $\mathrm{CA}$, Morais AB, Marinho PE. Whole-body vibration improves functional capacity and quality of life in patients with severe chronic obstructive pulmonary disease (COPD): a pilot study. Int J Chron Obstruct Pulmon Dis 2015;10:125-132.

32. Kenn K, Gloeckl R, Soennichsen A, Sczepanski B, Winterkamp S, Boensch M, Welte T. Predictors of success for pulmonary rehabilitation in patients awaiting lung transplantation. Transplantation 2015; 99(5):1072-1077.

33. Greulich T, Kehr K, Nell C, Koepke J, Haid D, Koehler U, et al. A randomized clinical trial to assess the influence of a three months training program (gym-based individualized vs. calisthenics-based non-invidualized) in COPD-patients. Respir Res 2014;15:36.

34. Porto EF, Castro AA, Schmidt VG, Rabelo HM, Kümpel C, Nascimento OA, Jardim JR. Postural control in chronic obstructive pulmonary disease: a systematic review. Int $\mathrm{J}$ Chron Obstruct Pulmon Dis 2015;10:1233-1239.

35. Kawanabe K, Kawashima A, Sashimoto I, Takeda T, Sato Y, Iwamoto $\mathrm{J}$. Effect of whole-body vibration exercise and muscle strengthening, balance, and walking exercises on walking ability in the elderly. Keio J Med 2007;56(1):28-33.

36. Rittweger J, Mutschelknauss M, Felsenberg D. Acute changes in neuromuscular excitability after exhaustive whole body vibration exercise as compared to exhaustion by squatting exercise. Clin Physiol Funct Imaging 2003;23(2):81-86.

37. Lamont HS, Cramer JT, Bemben DA, Shehab RL, Anderson MA, Bemben MG. Effects of adding whole body vibration to squat training on isometric force/time characteristics. J Strength Cond Res 2010;24(1):171-183.

38. Bosco C, Colli R, Introini E, Cardinale M, Tsarpela O, Madella A, et al. Adaptive responses of human skeletal muscle to vibration exposure. Clin Physiol 1999;19(2):183-187.

39. Ritzmann R, Gollhofer A, Kramer A. The influence of vibration type, frequency, body position and additional load on the neuromuscular activity during whole body vibration. Eur J Appl Physiol 2013; 113(1):1-11.

40. Ghobadi H, Ahari SS, Kameli A, Lari SM. The relationship between COPD Assessment Test (CAT) scores and severity of airflow obstruction in stable COPD patients. Tanaffos 2012;11(2):22-26.

41. Jones PW. St. George's Respiratory Questionnaire: MCID. COPD 2005;2(1):75-79. 